Journal of Advanced Computer Science \& Technology, 9 (1) (2020) 6-10
Sournal of Advanced Computer Science \& Technology
WPC
Website: www.sciencepubco.com/index.php/JACST
Research paper

\title{
Statistical adjustment of the parameters of multi-objective optimization problems with design expert method
}

\author{
Atefeh Hasan-Zadeh * \\ Fouman Faculty of Engineering, College of Engineering, University of Tehran, Iran \\ *Corresponding author E-mail: hasanzadeh.a@ut.ac.ir
}

\begin{abstract}
Optimization methods in which one single criterion is considered cannot provide a comprehensive solution to various decision- making algorithms because they cannot consider the interchange of conflicting goals that sometimes conflict with one another. Multi-objective opti-mization is a suitable solution to this obstacle. Given the importance of multi-objective optimization problems in engineering and technology, adjusting the parameters of these types of problems will, in addition to the decision-making accuracy, facilitate the analysis of the results and makes it more applicable. For this purpose, multi-objective optimization using experimental design methods has been developed which can solve these problems by considering different objectives simultaneously. Mathematical modelling for the setting of the parameters of the considered problem with all the statistical details related to their prediction and optimization have been studied.
\end{abstract}

Keywords: Statistical Modelling; Multi-Objective Optimization Problem; Adjustment of the Parameters; Design Expert Method.

\section{Introduction}

In science and technology, many of the problems involve several goals with constraints that combine these goals. Multi-objective optimization (or Pareto optimization) is a multi-criteria decision-making field that addresses these problems of mathematical optimization, and, it involves more than one target function that is simultaneously optimized. This type of optimization has been used in many fields of science and engineering in which desirable decisions should be made in spite of differences between two or more conflicting goals.

For process optimization in engineering and science, many problems include some goals that cannot be described as "better" or "lessbetter", instead, there is an ideal target value for each objective, and the desire to maximize the desired value of each objective [1-4]. For a multi-objective optimization problem, there's no solution to simultaneously optimize each objective which causes the researchers to examine these problems from different perspectives. Often such problems create linear line constraints that prevent all goals being simultaneously fully met, especially when the number of controlled variables is less than the number of targets and the time the random shocks occur, which is uncertain.

Typically, a multifunctional quantum objective function is used, with a cost that increases with a target, the fourth is far from the target of its ideal value. Since these problems usually involve adjusting variables controlled at different points in time and/or evaluating targets at different points in time, intertemporal optimization methods are used [5-7].

In the sequel, a general economic multi-objective optimization problem for adjusting its parameters is discussed. All statistical details related to modelling and optimization of the problem are described in detail. Numerous figures and tables are provided for this purpose.

\section{Statement of the problem}

Consumer demand for different goods is determined by the process of maximizing the services provided by these commodities and is limited by the amount of income available for consumer goods and the price of goods. This limitation allows more than one product to be purchased only for sacrificing consumption of less than one other product; therefore, different goals (consuming more each of the preferred products) differ.

Product and process design can be greatly improved by using modernization, simulation and optimization techniques [8-11]. Before looking for optimal designs, it is important to identify the features that have the highest overall design value. Therefore, in practical applications, process performance and product design are often measured concerning multiple objectives.

Considering a standardized multivariate optimization problem in economics and its usual factors such as NSP, MID, DM, and QM, mathematical modelling and statistical optimization for adjusting these parameters will be presented in the sequel. It should be noted that the invoiced factors are respectively indicated network and service performance, manufacturing ideal performance, demand for manufacturing, and Quality corporate manufacturing strategy. 


\section{Methodology}

All paragraphs must be justified alignment. With justified alignment, both sides of the paragraph are straight.

Statistical modelling is performed using the method of Historical Data as one of the response surface methodology techniques of design expert methods to determine the mathematical relationship between the problem variables and the objective function.

The top and bottom levels of each of the input variables and the output variables as well as other statistical information are summarized in Tables 1 and 2 .

Table 1: Design Summary: Inputs

\begin{tabular}{lllllllll}
\hline Factor Coded & Name & Type & Low Actual & High Actual & Low Coded & High Coded & Mean & Std. Dev. \\
\hline A & NSP & Numeric & 0 & 80 & -1 & 1 & 42.51852 & 22.73579 \\
B & MID & Numeric & 0 & 45.09804 & -1 & 1 & 21.20552 \\
C & DM & Numeric & 0 & 33.63636 & -1 & 1 & 18.53213 \\
D & QM & Numeric & 0 & 90.76428 & -1 & 1 & 52.04986 & 10.46613 \\
\hline
\end{tabular}

Table 2: Design Summary: Output

\begin{tabular}{llllllll}
\hline Response & Obs & Analysis & Minimum & Maximum & Mean & Std. Dev. & Ratio \\
\hline $\mathrm{R}$ & 27 & Polynomial & 11.58736 & 62.1274 & 37.09468 & 12.34014 & 5.361652 \\
\hline
\end{tabular}

For the multiple criteria decision making, a multi-objective optimization problem studies is expressed as a mathematical optimization problem which has been reduced to single-objective problem with introducing the weighted mean between factors as Equation 1:

$$
R=(N S P+2 M I D+D M+3 Q M) / 7
$$

These weights are based on the importance of the factors in the removal process. Of course, there are other methods, such as the estimation of entropy, to determine the weight of each factor in the multi-objective decision problems, which is not discussed here, and more details can be found in [12-13]. Different layouts of the four input variables are considered in the experiment.

The corresponding responses for each experiment were obtained according to formula 1 and are presented in Table 3.

\begin{tabular}{|c|c|c|c|c|c|c|c|c|c|c|c|}
\hline Run & NSP & MID & DM & $\mathrm{QM}$ & $\mathrm{R}$ & Run & NSP & MID & $\mathrm{DM}$ & $\mathrm{QM}$ & $\mathrm{R}$ \\
\hline 1 & 24 & 44.12 & 26.36 & 64.03 & 47.24 & 15 & 40 & 20.59 & 24.55 & 0.00 & 15.10 \\
\hline 2 & 64 & 5.88 & 12.73 & 63.35 & 39.79 & 16 & 16 & 0.00 & 22.73 & 72.40 & 36.56 \\
\hline 3 & 24 & 40.20 & 24.55 & 20.24 & 27.09 & 17 & 28 & 8.82 & 10.00 & 52.19 & 30.32 \\
\hline 4 & 60 & 0.00 & 27.27 & 57.72 & 37.20 & 18 & 68 & 17.65 & 24.55 & 74.62 & 50.24 \\
\hline 5 & 48 & 23.53 & 0.00 & 67.08 & 42.33 & 19 & 32 & 14.71 & 15.45 & 86.09 & 47.87 \\
\hline 6 & 16 & 4.90 & 5.45 & 85.79 & 41.23 & 20 & 56 & 26.47 & 18.18 & 70.53 & 48.39 \\
\hline 7 & 72 & 44.12 & 16.36 & 78.50 & 58.87 & 21 & 16 & 43.14 & 6.36 & 32.88 & 29.61 \\
\hline 8 & 16 & 13.73 & 2.73 & 25.34 & 17.46 & 22 & 56 & 45.10 & 21.82 & 39.65 & 41.00 \\
\hline 9 & 80 & 5.88 & 25.45 & 47.95 & 37.29 & 23 & 0 & 27.45 & 27.27 & 87.67 & 49.31 \\
\hline 10 & 24 & 14.71 & 28.18 & 50.85 & 33.45 & 24 & 44 & 35.29 & 0.00 & 46.74 & 36.40 \\
\hline 11 & 64 & 14.71 & 8.18 & 13.78 & 20.42 & 25 & 72 & 7.84 & 26.36 & 66.66 & 44.86 \\
\hline 12 & 8 & 15.69 & 33.64 & 2.70 & 11.59 & 26 & 68 & 8.82 & 30.91 & 40.14 & 33.85 \\
\hline 13 & 48 & 44.12 & 26.36 & 90.76 & 62.13 & 27 & 68 & 15.69 & 33.64 & 10.25 & 23.39 \\
\hline 14 & 36 & 29.41 & 2.73 & 57.39 & 38.53 & & & & & & \\
\hline
\end{tabular}

For the input-output data set, different models were fitted which were the best linear equation since for all the appropriate conditions for statistical analysis. The most important of these are:

P-value $<0.0001$

Adjusted R-Squared: 1.000

Predicted R-Squared: 1.000

Highest order polynomial where the additional terms are significant and the model is not aliased which represent the fully acceptable validity of the fitted model. Variance analysis was then performed on the best models to evaluate their accuracy, the results of which can be seen in Table 4.

Table 4: Analysis of Variance (ANOVA) for Response Surface Quadratic Model

\begin{tabular}{llllll}
\multicolumn{5}{c}{ Table 4: Analysis of ariance (ANOVA) for Response Surface Quadratic Model } \\
\hline Source & Sum of Squares & df & Mean Square & F Value & p-value Prob $>$ F \\
\hline Model & 4111.533 & 4 & 1027.883 & 63660000 & $<0.0001$ \\
A-NPS & 275.4668 & 1 & 275.4668 & 63660000 & $<0.0001$ \\
B-MID & 455.4064 & 1 & 455.4064 & 63660000 & $<0.0001$ \\
C-DM & 57.72235 & 1 & 57.72235 & 63660000 & $<0.0001$ \\
D-QM & 3359.906 & 1 & 3359.906 & 63660000 & $<0.0001$ \\
Residual & 0 & 22 & 0 & & \\
Cor Total & 4111.533 & 26 & & & \\
\hline
\end{tabular}

Table 4 shows that the fitted model is reliable with a confidence interval of $99.9 \%$ because the Prob>F parameter is less than 0.0001 . As such, the set of the obtained statistical parameters indicates the high accuracy and reliability of the fitted model, which can be used as an objective function for optimization.

Also, the final equation in terms of actual factors is following equation (2): 
Comparison between the actual value and predicted value, are depicted in Figures 1 and 2. Fig. 1 and 2 show the non-discrepancy between the predicted values and the actual values.

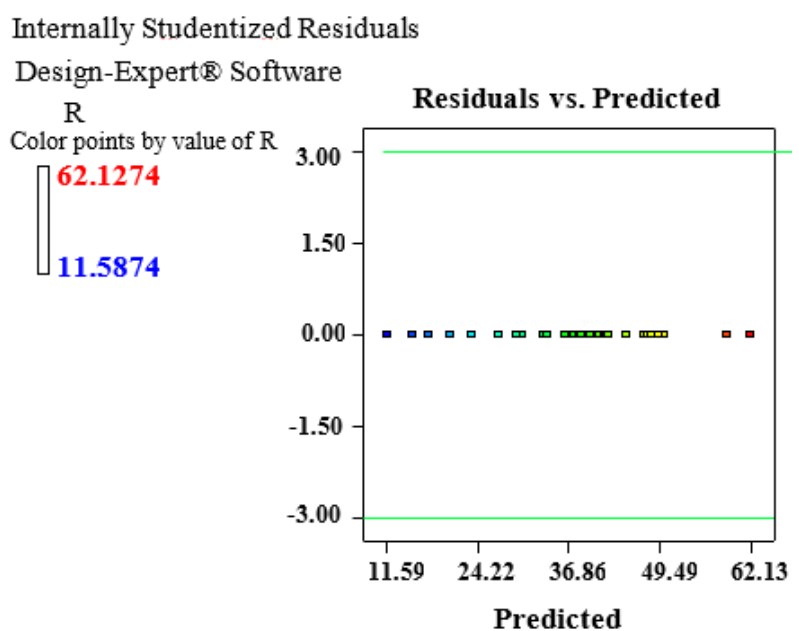

Fig. 1: Residuals vs. Predicted.

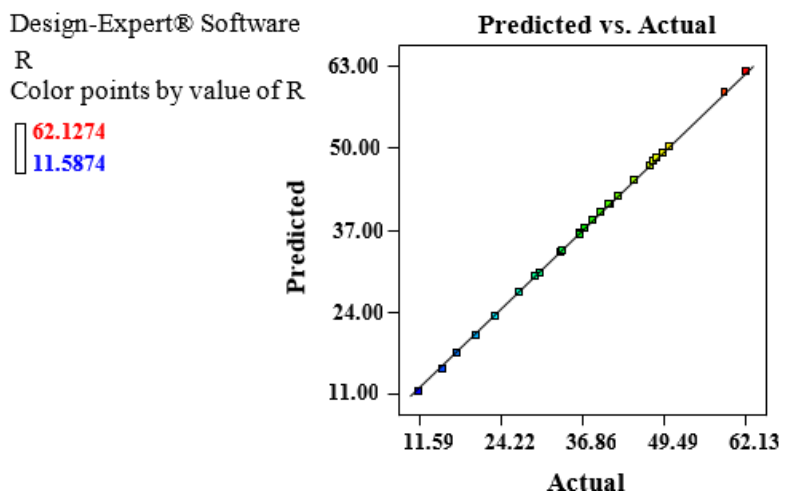

Fig. 2: Predicted vs. Actual.

Mathematical modelling is plotted in Fig. 3.

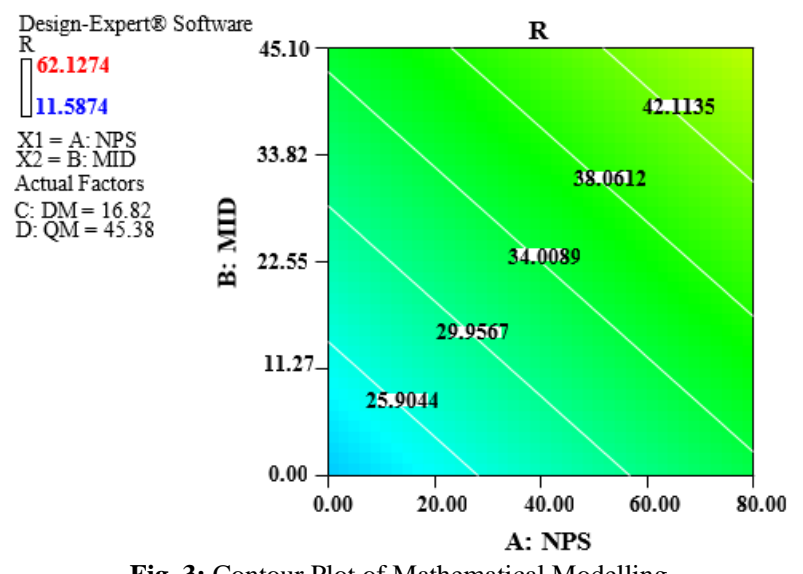

Fig. 3: Contour Plot of Mathematical Modelling.

Finally, the optimal response to the maximum efficiency is given in the following table.

Table 6: Optimization Solutions

\begin{tabular}{|c|c|c|c|c|c|c|c|c|c|c|c|c|c|}
\hline Number & NPS & MID & $\mathrm{DM}$ & QM & $\mathrm{R}$ & Desirability & Number & NPS & MID & DM & QM & $\mathrm{R}$ & Desirability \\
\hline 1 & 79.96 & 42.81 & 9.49 & 88.52 & 62.94595 & 1 & 16 & 66.72 & 43.23 & 12.7 & 90.56 & 62.50895 & 1 \\
\hline 2 & 68.78 & 36.18 & 31.28 & 90.64 & 63.47499 & 1 & 17 & 75.57 & 43.63 & 22.18 & 83.82 & 62.35313 & 1 \\
\hline 3 & 78.38 & 35.47 & 18.94 & 90.11 & 62.65631 & 1 & 18 & 79.93 & 32.55 & 18.3 & 90.74 & 62.21986 & 1 \\
\hline 4 & 48.99 & 43.43 & 31.3 & 89.47 & 62.22306 & 1 & 19 & 70.23 & 38.96 & 17.34 & 90.11 & 62.25636 & 1 \\
\hline 5 & 73.46 & 33.67 & 23.57 & 90.5 & 62.26771 & 1 & 20 & 74.44 & 44.83 & 33.37 & 86.23 & 65.16768 & 1 \\
\hline 6 & 79.97 & 42.34 & 22.59 & 83.4 & 62.48926 & 1 & 21 & 78.27 & 44.84 & 2.25 & 90.53 & 63.11146 & 1 \\
\hline 7 & 77.56 & 42.64 & 10.08 & 87.95 & 62.39507 & 1 & 22 & 67.89 & 41.8 & 26.54 & 90.75 & 64.32423 & 1 \\
\hline 8 & 66.41 & 44.24 & 27.29 & 89.55 & 64.40329 & 1 & 23 & 78.36 & 44.53 & 11.02 & 89.61 & 63.89371 & 1 \\
\hline 9 & 68.53 & 41.61 & 33.08 & 89.95 & 64.95464 & 1 & 24 & 66.8 & 45.1 & 0 & 90.76 & 61.32711 & 0.984165 \\
\hline 10 & 49.29 & 44.77 & 26.59 & 90.67 & 62.49024 & 1 & 25 & 31.82 & 42.79 & 33.64 & 90.76 & 60.47394 & 0.967284 \\
\hline 11 & 76.83 & 39.17 & 24.27 & 90.47 & 64.40593 & 1 & 26 & 80 & 32.8 & 2.81 & 90.76 & 60.10083 & 0.959902 \\
\hline
\end{tabular}




\begin{tabular}{|c|c|c|c|c|c|c|c|c|c|c|c|c|c|}
\hline 12 & 77.44 & 44.16 & 2.12 & 89.92 & 62.52073 & 1 & 27 & 55.77 & 45.1 & 0.02 & 90.68 & 59.71777 & 0.952322 \\
\hline 13 & 65.38 & 44.64 & 12.58 & 90.56 & 62.70084 & 1 & 28 & 54.67 & 45.1 & 0.02 & 90.76 & 59.59679 & 0.949929 \\
\hline 14 & 70.39 & 38.66 & 31.71 & 89.02 & 63.7843 & 1 & 29 & 27.28 & 45.1 & 16.21 & 90.76 & 57.99777 & 0.91829 \\
\hline 15 & 67.63 & 42.46 & 30.92 & 89.17 & 64.42663 & 1 & 30 & 47.01 & 17.67 & 33.64 & 90.76 & 55.46874 & 0.86825 \\
\hline
\end{tabular}

For example the statistical plots for the first optimum solution are shown in Fig. 4 and 5.

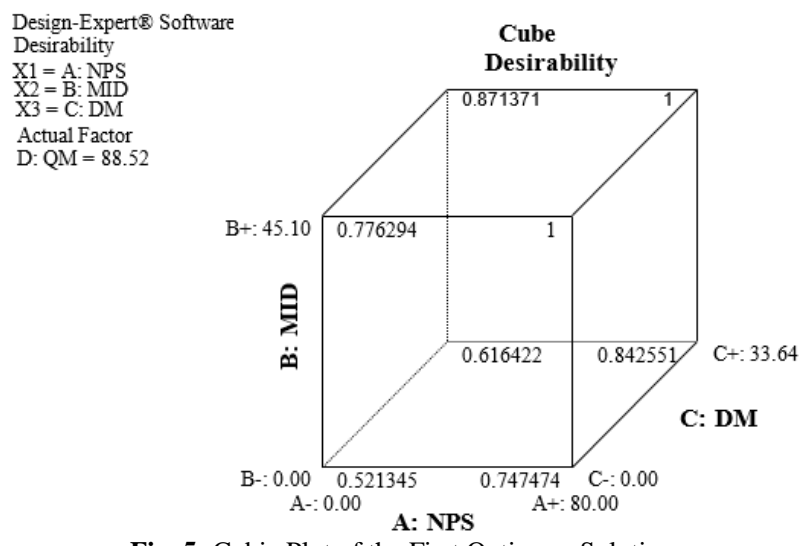

Fig. 5: Cubic Plot of the First Optimum Solution.

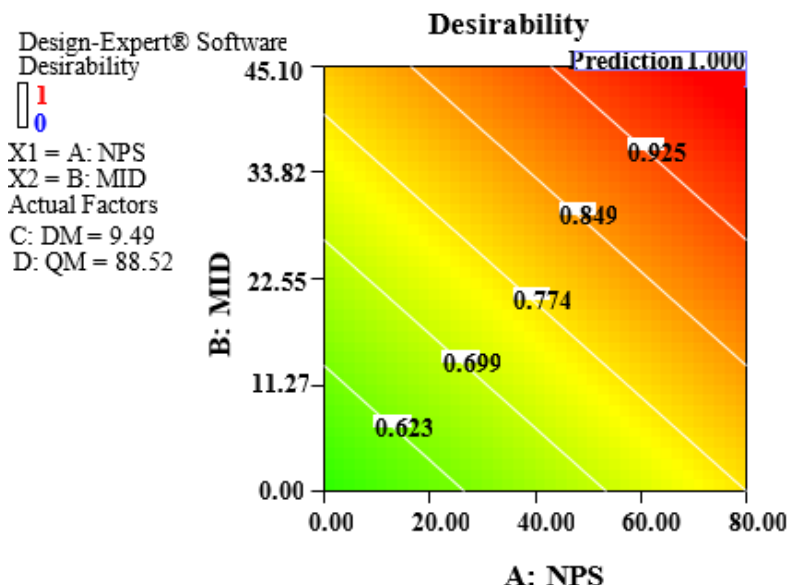

Fig. 6: Contour Plot of the First Optimum Solution.

\section{Conclusion}

The parameters of a general economic multi-objective optimization problem were adjusted. To this end, the problem under consideration was transformed into a single-objective problem by considering the weight of each of its parameters. Mathematical modelling was performed to determine the statistical relationship between inputs and the objective of the problem. Prediction of parameter values was performed to maximize the single-objective function. All possible choices for the parameters were provided for the optimal target. The high accuracy of the analysis was shown statistically using the figures and tables.

\section{References}

[1] Domingo-Perez F, Lazaro-Galilea, JL, Wieser A, Martin-Gorostiza E, Salido-Monzu D \& de la Llana A (2016), Sensor placement determination for range-difference positioning using evolutionary multi-objective optimization, Expert Systems with Applications, 47(1), 95-105. https://doi.org/10.1016/j.eswa.2015.11.008.

[2] Ghaithan AM, Attia A \& Duffuaa, SO, (2017), Multi-objective optimization model for a downstream oil and gas supply chain, Applied Mathematical Modelling, 52, 689-708. https://doi.org/10.1016/j.apm.2017.08.007.

[3] Hasan-Zadeh A, (2019), Geometric Modelling of the Thinning by Cell Complexes, Journal of Advanced Computer Science \& Technology, 8(2), 3839.

[4] Hasan-Zadeh A, (2019), Mathematical Modelling of Decision-Making: Application to Investment, Advances in Decision Sciences, $23(2), 1-14$.

[5] Ghobadi-Nejad Z, Borghei SM, Yaghmaei S \& Hasan-Zadeh A, (2019), Developing a new approach for (biological) optimal control problems: application to optimization of laccase production with a comparison between response surface methodology and novel geometric procedure, Mathematical Biosciences, 309, 23-33. https://doi.org/10.1016/j.mbs.2018.12.013.

[6] Samadi A, Sharifi H, Ghobadi-Nejad Z, Hasan-Zadeh A \& Yaghmaei, S, (2020), Biodegradation of 4-Chlorobenzoic Acid by Lysinibacillus macrolides DSM54T and Determination of Optimal Conditions, International Journal of Environmental Research, 15(1), 1-10. https://doi.org/10.1007/s41742-020-00247-4.

[7] Ghasemi S, Mirzaie M, Hasan-Zadeh A, Ashrafnezhad M, Hashemian SJ \& Shahnemati SR, Design, operation, performance evaluation and mathematical optimization of a vermifiltration pilot plan for domestic wastewater treatment, Journal of Environmental Chemical Engineering, 8(1), 103587. https://doi.org/10.1016/j.jece.2019.103587.

[8] Amirahmadi A, Dastfan A \& Rafiei SMR, (2012), Optimal Controller Design for Single-phase PWM Rectifier Using SPEA Multi-objective Optimization, Journal of Power Electronics, 12(1), 104-112. https://doi.org/10.6113/JPE.2012.12.1.104.

[9] Rafiei SMR, Amirahmadi A \& Griva G, (2009), Chaos Rejection and Optimal Dynamic Response for Boost Converter Using SPEA Multi-Objective Optimization Approach, IEEE Iecon, 3351-3358. https://doi.org/10.1109/IECON.2009.5415056. 
[10] Ganesan T, Elamvazuthi I, Ku Shaari KZ \& Vasant P, (2013), Swarm intelligence and gravitational search algorithm for multi-objective optimization of synthesis gas production, Applied Energy, 103(1), 368-374. https://doi.org/10.1016/j.apenergy.2012.09.059.

[11] Ganesan T, Elamvazuthi I, Vasant P, \& Ku Shaari KZ, Nguyen, N. T., Trawiński, B., Kosala, R., (eds.), Multiobjective Optimization of Bioactive Compound Extraction Process via Evolutionary Strategies, Lecture Notes in Computer Science, Springer International Publishing, 2015. https://doi.org/10.1007/978-3-319-15705-4_2.

[12] Pearce M, Mutlu B, Shah J, Radwin R, (2018), Optimizing Makespan and Ergonomics in Integrating Collaborative Robots into Manufacturing Processes, IEEE Transactions on Automation Science and Engineering, 15(4), 1772-1784. https://doi.org/10.1109/TASE.2018.2789820.

[13] Lobato FS \& Steffen J, Multi-Objective Optimization Problems: Concepts and Self-Adaptive Parameters with Mathematical and Engineering Applications, Springer, 2017. https://doi.org/10.1007/978-3-319-58565-9. 